\title{
POSITIVE GRASSMANNIAN AND POLYHEDRAL SUBDIVISIONS
}

\author{
ALEXANDER POSTNIKOV
}

\begin{abstract}
The nonnegative Grassmannian is a cell complex with rich geometric, algebraic, and combinatorial structures. Its study involves interesting combinatorial objects, such as positroids and plabic graphs. Remarkably, the same combinatorial structures appeared in many other areas of mathematics and physics, e.g., in the study of cluster algebras, scattering amplitudes, and solitons. We discuss new ways to think about these structures. In particular, we identify plabic graphs and more general Grassmannian graphs with polyhedral subdivisions induced by 2 -dimensional projections of hypersimplices. This implies a close relationship between the positive Grassmannian and the theory of fiber polytopes and the generalized Baues problem. This suggests natural extensions of objects related to the positive Grassmannian.
\end{abstract}

\section{INTRODUCTION}

The geometry of the Grassmannian $\operatorname{Gr}(k, n)$ is related to combinatorics of the hypersimplex $\Delta_{k n}$. Gelfand, Goresky, MacPherson, Serganova GGMS87 studied the hypersimplex as the moment polytope for the torus action on the complex Grassmannian. In this paper we highlight new links between geometry of the positive Grassmannian and combinatorics of the hypersimplex $\Delta_{k n}$.

GGMS87 studied the matroid stratification of the Grassmannian $\operatorname{Gr}(k, n, \mathbb{C})$, whose strata are the realization spaces of matroids. They correspond to matroid polytopes living inside $\Delta_{k n}$. In general, matroid strata are not cells. In fact, according to Mnëv's universality theorem Mnë88, the matroid strata can be as complicated as any algebraic variety. Thus the matroid stratification of the Grassmannian can have arbitrarily bad behavior.

There is, however, a semialgebraic subset of the real Grassmannian $\operatorname{Gr}(k, n, \mathbb{R})$, called the nonnegative Grassmannian $G r \geq 0(k, n)$, where the matroid stratification exhibits a well behaved combinatorial and geometric structure. Its structure, which is quite rich and nontrivial, can nevertheless be described in explicit terms. In some way, the nonnegative Grassmannian is similar to a polytope.

The notion of a totally positive matrix, that is a matrix with all positive minors, originated in pioneering works of Gantmacher, Krein GK35, and Schoenberg Sch30. Since then such matrices appeared in many areas of pure and applied mathematics. Lusztig [Lus94, Lus98a, Lus98b] generalized the theory of total positivity

Date: February 27, 2018.

2010 Mathematics Subject Classification. Primary 05E; secondary 52B, 52C, 13F60, 81T.

Key words and phrases. Total positivity, positive Grassmannian, hypersimplex, matroids, positroids, cyclic shifts, Grassmannian graphs, plabic graphs, polyhedral subdivisions, triangulations, zonotopal tilings, associahedron, fiber polytopes, Baues poset, generalized Baues problem, flips, cluster algebras, weakly separated collections, scattering amplitudes, amplituhedron, membranes. 
in the general context of Lie theory. He defined the positive part for a reductive Lie group $G$ and a generalized flag variety $G / P$. Rietsch [Rie98, Rie99] studied its cellular decomposition. Lusztig's theory of total positivity has close links with his theory of canonical bases Lus90, Lus92, Lus93 and Fomin-Zelevinsky's cluster algebras [FZ02a, FZ02b, FZ03, BFZ05, FZ07.

[Pos06] initiated a combinatorial approach to the study of the positive Grassmannian. The positive (resp., nonnegative) Grassmannian $G r^{>0}(k, n)(G r \geq 0(k, n))$ was described as the subset of the Grassmannian $\operatorname{Gr}(k, n, \mathbb{R})$ where all Plücker coordinates are positive (resp., nonnegative). This "elementary" definition agrees with Lusztig's general notion Lus98a] of the positive part of $G / P$ in the case when $G / P=G r(k, n)$.

The positroid cells, defined as the parts of matroid strata inside the nonnegative Grassmannian, turned out to be indeed cells. (The term "positroid" is an abbreviation for "positive matroid.") The positroid cells form a CW-complex. Conjecturally, it is a regular CW-complex, and the closure of each positroid cell is homeomorphic to a closed ball. This positroid stratification of $G r^{\geq 0}(k, n)$ is the common refinement of $n$ cyclically shifted Schubert decompositions Pos06. Compare this with the result GGMS87 that the matroid stratification of $G r(k, n)$ is the common refinement of $n$ ! permuted Schubert decompositions. The cyclic shift plays a crucial role in the study of the positive Grassmannian. Many objects associated with the positive Grassmannian exhibit cyclic symmetry.

Positroid cells were identified in Pos06] with many combinatorial objects, such as decorated permutation, Grassmann necklaces, etc. Moreover, an explicit birational subtraction-free parametrization of each cell was described in terms of plabic graphs, that is, planar bicolored graphs, which are certain graphs embedded in a disk with vertices colored in two colors.

Remarkably, the combinatorial structures that appeared in the study of the positive Grassmannian also surfaced and played an important role in many different areas of mathematics and physics. Scott [Sco05, Sco06] and OPS15] linked these objects with cluster algebra structure on the Grassmannian and with LeclercZelevinsky's quasi-commutting families of quantum minors and weakly separated collections. Corteel and Williams CW07] applied Le-diagrams (which correspond to positroids) to the study of the partially asymmetric exclusion process (PASEP). Knutson, Lam, and Speyer KLS13 proved that the cohomology classes of the positroid varieties (the complexifications of the positroid cells) are given by the affine Stanley symmetric functions, which are dual to Lapointe-Lascoux-Morse $k$ Schur functions. They also linked positroids with theory of juggling. Plabic graphs appeared in works of Chakravarty, Kodama, and Williams [CK09, KoW11, KoW14] as soliton solutions of the Kadomtsev-Petviashvili (KP) equation, which describes nonlinear waves. Last but not least, plabic graphs appeared under the name of on-shell diagrams in the work by Arkani-Hamed et al [ABCGPT16] on scattering amplitudes in $\mathcal{N}=4$ supersymmetric Yang-Mills (SYM) theory. They play a role somewhat similar to Feynman diagrams, however, unlike Feynman diagrams, they represent on-shell processes and do not require introduction of virtual particles.

In this paper, we review some of the main constructions and results from Pos06. PSW09, OPS15] related to the positive Grassmannian. We extend these constructions in the language of Grassmannian graphs. The parametrization of a positroid cell in $G r^{\geq 0}(k, n)$ given by a Grassmannian graph can be thought of as a way to 
"glue" the positroid cell out of "little positive Grassmannians" associated with vertices of the graph. The idea to think about parametrizations of cells as gluings of Grassmannians came originally from physics [ABCGPT16, where vertices of onshell diagrams (i.e., plabic graphs) were viewed as little Grassmannians $\operatorname{Gr}(1,3)$ and $\operatorname{Gr}(2,3)$.

We link this construction of parametrizations of $G r^{>0}(k, n)$ given by Grassmannian graphs with the study of polyhedral subdivisions induced by 2-dimensional cyclic projections $\pi: \Delta_{k n} \rightarrow Q$ of the hypersimplex. Reduced Grassmannian graphs parametrizing the positive Grassmannian $G r^{>0}(k, n)$ turn out to be in bijection with $\pi$-induced polyhedral subdivisions. Thus gluing of Grassmannians from smaller Grassmannians is equivalent to subdividing polytopes into smaller polytopes. The study of $\pi$-induced subdivisions for projections of polytopes is the subject of Billera-Sturmfels' theory BS92 of fiber polytopes and the generalized Baues problem (GBP) posed by Billera, Kapranov, Sturmfels BKS94. We also mention the result of Galashin Gal16 where plabic graphs are identified with sections of zonotopal tilings, and the construction from the joint work $[\mathrm{LP}]$ with Lam on polypositroids where plabic graphs are viewed as membranes, which are certain 2-dimensional surfaces in higher dimensional spaces.

The correspondence between parametrizations of the positive Grassmannian and polyhedral subdivisions leads to natural generalizations and conjectures. We discuss a possible extension of constructions of this paper to "higher positive Grassmannians" and amplituhedra of Arkani-Hamed and Trnka [AT14].

I thank Federico Ardila, Nima Arkani-Hamed, Arkady Berenstein, David Bernstein, Lou Billera, Jacob Bourjaily, Freddy Cachazo, Miriam Farber, Sergey Fomin, Pavel Galashin, Israel Moiseevich Gelfand, Oleg Gleizer, Alexander Goncharov, Darij Grinberg, Alberto Grünbaum, Xuhua He, Sam Hopkins, David Ingerman, Tamás Kálmán, Mikhail Kapranov, Askold Khovanskii, Anatol Kirillov, Allen Knutson, Gleb Koshevoy, Thomas Lam, Joel Lewis, Gaku Liu, Ricky Liu, George Lusztig, Thomas McConville, Karola Mészáros, Alejandro Morales, Gleb Nenashev, Suho Oh, Jim Propp, Pavlo Pylyavskyy, Vic Reiner, Vladimir Retakh, Konni Rietsch, Tom Roby, Yuval Roichman, Paco Santos, Jeanne Scott, Boris Shapiro, Michael Shapiro, David Speyer, Richard Stanley, Bernd Sturmfels, Dylan Thurston, Jaroslav Trnka, Wuttisak Trongsiriwat, Vladimir Voevodsky, Lauren Williams, Hwanchul Yoo, Andrei Zelevinsky, and Günter Ziegler for insightful conversations. These people made a tremendous contribution to the study of the positive Grassmannian and related combinatorial, algebraic, geometric, topological, and physical structures. Many themes we discuss here are from past and future projects with various subsets of these people.

\section{Grassmannian And matroids}

Fix integers $0 \leq k \leq n$. Let $[n]:=\{1, \ldots, n\}$ and $\left(\begin{array}{c}{[n]} \\ k\end{array}\right)$ be the set of $k$-element subsets of $[n]$.

The Grassmannian $\operatorname{Gr}(k, n)=\operatorname{Gr}(k, n, \mathbb{F})$ over a field $\mathbb{F}$ is the variety of $k$ dimensional linear subspaces in $\mathbb{F}^{n}$. More concretely, $G r(k, n)$ is the space of $k \times n$ matrices of rank $k$ modulo the left action of $G L(k)=G L(k, \mathbb{F})$. Let $[A]=G L(k) A$ be the element of $\operatorname{Gr}(k, n)$ represented by matrix $A$. 
Maximal minors $\Delta_{I}(A)$ of such matrices $A$, where $I \in\left(\begin{array}{c}{[n]} \\ k\end{array}\right)$, form projective coordinates on $\operatorname{Gr}(k, n)$, called the Plücker coordinates. For $[A] \in G r(k, n)$, let

$$
\mathcal{M}(A):=\left\{I \in\left(\begin{array}{c}
{[n]} \\
k
\end{array}\right) \mid \Delta_{I}(A) \neq 0\right\} .
$$

The sets of the form $\mathcal{M}(A)$ are a special kind of matroids, called $\mathbb{F}$-realizable matroids. Matroid strata are the realization spaces of realizable matroids $\mathcal{M} \subset\left(\begin{array}{c}{[n]} \\ k\end{array}\right)$ :

$$
S_{\mathcal{M}}:=\{[A] \in \operatorname{Gr}(k, n) \mid \mathcal{M}(A)=\mathcal{M}\} .
$$

The matroid stratification is the disjoint decomposition

$$
G r(k, n)=\bigsqcup_{\mathcal{M} \text { realizable matroid }} S_{\mathcal{M}}
$$

The Gale order " $\preceq$ " (or the coordinatewise order) is the partial order on $\left(\begin{array}{c}{[n]} \\ k\end{array}\right)$ given by $\left\{i_{1}<\cdots<i_{k}\right\} \preceq\left\{j_{1}<\cdots<j_{k}\right\}$, if $i_{r} \leq j_{r}$ for $r \in[k]$. Each matroid $\mathcal{M}$ has a unique minimal element $I_{\min }(\mathcal{M})$ with respect to the Gale order.

For $I \in\left(\begin{array}{c}{[n]} \\ k\end{array}\right)$, the Schubert cell $\Omega_{I} \subset G r(k, n)$ is given by

$$
\Omega_{I}:=\left\{[A] \in G r(k, n) \mid I=I_{\min }(\mathcal{M}(A)\}=\bigsqcup_{\mathcal{M}: I=I_{\min }(\mathcal{M})} S_{\mathcal{M}} .\right.
$$

They form the Schubert decomposition $\operatorname{Gr}(k, n)=\bigsqcup \Omega_{I}$. Clearly, for a realizable matroid $\mathcal{M}$, we have $S_{\mathcal{M}} \subset \Omega_{I}$ if and only if $I=I_{\min }(\mathcal{M})$.

The symmetric group $S_{n}$ acts on $G r(k, n)$ by permutations $w\left(\left[v_{1}, \ldots, v_{n}\right]\right)=$ $\left[v_{w(1)}, \ldots, v_{w(n)}\right]$ of columns of $[A]=\left[v_{1}, \ldots, v_{n}\right] \in G r(k, n)$.

It is clear that, see GGMS87, the matroid stratification of $\operatorname{Gr}(k, n)$ is the common refinement of the $n$ ! permuted Schubert decompositions. In other words, each matroid stratum $S_{\mathcal{M}}$ is an intersection of permuted Schubert cells:

$$
S_{\mathcal{M}}=\bigcap_{w \in S_{n}} w\left(\Omega_{I_{w}}\right)
$$

Indeed, if we know the minimal elements of a set $\mathcal{M} \subset\left(\begin{array}{c}{[n]} \\ k\end{array}\right)$ with respect to all $n$ ! orderings of $[n]$, we know the set $\mathcal{M}$ itself.

\section{Positive Grassmannian And Positroids}

Fix the field $\mathbb{F}=\mathbb{R}$. Let $G r(k, n)=G r(k, n, \mathbb{R})$ be the real Grassmannian.

Definition 3.1. Pos06, Definition 3.1] The positive Grassmannian $\operatorname{Gr}^{>0}(k, n)$ (resp., nonnegative Grassmannian $G_{r} \geq 0(k, n)$ ) is the semialgebraic set of elements $[A] \in G r(k, n)$ represented by $k \times n$ matrices $A$ with all positive maximal minors $\Delta_{I}(A)>0$ (resp., all nonnegative maximal minors $\Delta_{I}(A) \geq 0$ ).

This definition agrees with Lusztig's general definition Lus98a of the positive part of a generalized flag variety $G / P$ in the case when $G / P=G r(k, n)$.

Definition 3.2. Pos06, Definition 3.2] A positroid cell $\Pi_{\mathcal{M}} \subset G r^{\geq 0}(k, n)$ is a nonempty intersection of a matroid stratum with the nonnegative Grassmannian:

$$
\Pi_{\mathcal{M}}:=S_{\mathcal{M}} \cap G r^{\geq 0}(k, n) .
$$


A positroid of rank $k$ is a collection $\mathcal{M} \subset\left(\begin{array}{c}{[n]} \\ k\end{array}\right)$ such that $\Pi_{\mathcal{M}}$ is nonempty. The positroid stratification of the nonnegative Grassmannian is the disjoint decomposition of $G r^{\geq 0}(k, n)$ into the positroid cells:

$$
G r^{\geq 0}(k, n)=\bigsqcup_{\mathcal{M} \text { is a positroid }} \Pi_{\mathcal{M}}
$$

Clearly, positroids, or positive matroids, are a special kind of matroids. The positive Grassmannian $G r^{>0}(k, n)$ itself is the top positroid cell $\Pi_{\left(\begin{array}{c}{[n]} \\ k\end{array}\right)}$ for the uniform matroid $\mathcal{M}=\left(\begin{array}{c}{[n]} \\ k\end{array}\right)$.

The cyclic shift is the map $\tilde{c}: G r(k, n) \rightarrow G r(k, n)$ acting on elements $[A]=$ $\left[v_{1}, \ldots, v_{n}\right] \in G r(k, n)$ by

$$
\tilde{c}:\left[v_{1}, \ldots, v_{n}\right] \longmapsto\left[v_{2}, v_{3}, \ldots, v_{n},(-1)^{k-1} v_{1}\right] .
$$

The shift $\tilde{c}$ induces the action of the cyclic group $\mathbb{Z} / n \mathbb{Z}$ on the Grassmannian $G r(k, n)$, that preserves its positive part $G r^{>0}(k, n)$. Many of the objects associated with the positive Grassmannian exhibit cyclic symmetry. This cyclic symmetry is a crucial ingredient in the study of the positive Grassmannian.

Theorem 3.3. Pos06, Theorem 3.7] The positroid stratification is the common refinement of $n$ cyclically shifted Schubert decompositions restricted to $G r{ }^{\geq 0}(k, n)$. In other words, each positroid cell $\Pi_{\mathcal{M}}$ is given by the intersection of the nonnegative parts of $n$ cyclically shifted Schubert cells:

$$
\Pi_{\mathcal{M}}=\bigcap_{i=0}^{n-1} \tilde{c}^{i}\left(\Omega_{I_{i}} \cap G r^{\geq 0}(k, n)\right) .
$$

So the positroid cells require intersecting $n$ cyclically shifted Schubert cells, which is a smaller number than $n$ ! permuted Schubert cells needed for general matroid strata. In fact, the positroid cells $\Pi_{\mathcal{M}}$ (unlike matroid strata) are indeed cells.

Theorem 3.4. Pos06, Theorem 3.5], PSW09, Theorem 5.4] The positroid cells $\Pi_{\mathcal{M}}$ are homeomorphic to open balls. The cell decomposition of $G r{ }^{\geq 0}(k, n)$ into the positroid cells $\Pi_{\mathcal{M}}$ is a $C W$-complex.

Conjecture 3.5. Pos06, Conjecture 3.6] The positroid stratification of the nonnegative Grassmannian $G r^{\geq 0}(k, n)$ is a regular $C W$-complex. In particular, the closure $\bar{\Pi}_{\mathcal{M}}$ of each positroid cell in $G r^{\geq 0}(k, n)$ is homeomorphic to a closed ball.

This conjecture was motivated by a similar conjecture of Fomin and Zelevinsky on double Bruhat cells [FZ99]. Up to homotopy-equivalence this conjecture was proved by Rietsch and Williams RW10. A major step towards this conjecture was recently achieved by Galashin, Karp, and Lam, who proved it for the top cell.

Theorem 3.6. GKL17, Theorem 1.1] The nonnegative Grassmannian $G r^{\geq 0}(k, n)$ is homeomorphic to a closed ball of dimension $k(n-k)$.

By Theorem 3.3 , positroids $\mathcal{M}$ and positroid cells $\Pi_{\mathcal{M}} \subset G r \geq 0(k, n)$ correspond to certain sequences $\left(I_{0}, I_{1}, \ldots, I_{n-1}\right)$. Let us describe this bijection explicitly.

Definition 3.7. Pos06, Definition 16.1] A Grassmann necklace $\mathcal{J}=\left(J_{1}, J_{2}, \ldots, J_{n}\right)$ of type $(k, n)$ is a sequence of elements $J_{i} \in\left(\begin{array}{c}{[n]} \\ k\end{array}\right)$ such that, for any $i \in[n]$, either $J_{i+1}=\left(J_{i} \backslash\{i\}\right) \cup\{j\}$ or $J_{i+1}=J_{i}$, where the indices $i$ are taken $(\bmod n)$. 
The cyclic permutation $c \in S_{n}$ is given by $c: i \mapsto i+1(\bmod n)$. The action of the symmetric group $S_{n}$ on $[n]$ induces the $S_{n}$-action on $\left(\begin{array}{c}{[n]} \\ k\end{array}\right)$ and on subsets of $\left(\begin{array}{c}{[n]} \\ k\end{array}\right)$. Recall that $I_{\min }(\mathcal{M})$ is the minimal element of a matroid $\mathcal{M}$ in the Gale order. For a matroid $\mathcal{M}$, let

$$
\begin{aligned}
& \mathcal{J}(\mathcal{M}):=\left(J_{1}, \ldots, J_{n}\right), \text { where } \\
& J_{i+1}=c^{i}\left(I_{\min }\left(c^{-i}(\mathcal{M})\right)\right), \text { for } i=0, \ldots, n-1 .
\end{aligned}
$$

Theorem 3.8. Pos06, Theorem 17.1] The map $\mathcal{M} \mapsto \mathcal{J}(\mathcal{M})$ is a bijection between positroids $\mathcal{M}$ of rank $k$ on the ground set $[n]$ and Grassmann necklaces of type $(k, n)$.

The sequence $\left(I_{0}, I_{1}, \ldots, I_{n-1}\right)$ associated with $\mathcal{M}$ as in Theorem 3.3 is related to the Grassmann necklace $\left(J_{1}, \ldots, J_{n}\right)$ of $\mathcal{M}$ by $I_{i}=c^{-i}\left(J_{i+1}\right)$, for $i=0, \ldots, n-1$.

The following result shows how to reconstruct a positroid $\mathcal{M}$ from its Grassmann necklace, cf. Theorem 3.3 For $I \in\left(\begin{array}{c}{[n]} \\ k\end{array}\right)$, the Schubert matroid is $\mathcal{M}_{I}:=\left\{J \in\left(\begin{array}{c}{[n]} \\ k\end{array}\right) \mid\right.$ $I \preceq J\}$, where "․" is the Gale order.

Theorem 3.9. Oh11, Theorem 6] For a Grassmann necklace $\mathcal{J}=\left(J_{1}, \ldots, J_{n}\right)$, the associated positroid $\mathcal{M}(\mathcal{J})=\mathcal{M}$ is given by

$$
\mathcal{M}=\bigcap_{i=0}^{n-1} c^{i}\left(\mathcal{M}_{I_{i}}\right)
$$

where $I_{i}=c^{-i}\left(J_{i+1}\right)$.

Let us describe positroids in the language of convex geometry. The hypersimplex

$$
\Delta_{k n}:=\operatorname{conv}\left\{e_{I} \mid I \in\left(\begin{array}{c}
{[n]} \\
k
\end{array}\right)\right\}
$$

is the convex hull of the $\left(\begin{array}{l}n \\ k\end{array}\right)$ points $e_{I}=\sum_{i \in I} e_{i}$, for all $I \in\left(\begin{array}{c}{[n]} \\ k\end{array}\right)$. Here $e_{1}, \ldots, e_{n}$ is the standard basis in $\mathbb{R}^{n}$. For a subset $\mathcal{M} \subset\left(\begin{array}{c}{[n]} \\ k\end{array}\right)$, let $P_{\mathcal{M}}:=\operatorname{conv}\left\{e_{I} \mid I \in \mathcal{M}\right\}$ be the convex hull of vertices of $\Delta_{k n}$ associated with elements of $\mathcal{M}$.

By GGMS87, $\mathcal{M}$ is a matroid if and only if every edge of the polytope $P_{\mathcal{M}}$ has the form $\left[e_{I}, e_{J}\right]$, for $I, J \in\left(\begin{array}{c}{[n]} \\ k\end{array}\right)$ with $|I \cap J|=k-1$. Here is an analogous description of positroids, which is not hard to derive from Theorem 3.9

Theorem 3.10. $\mathrm{LP}$ A nonempty subset $\mathcal{M} \subset\left(\begin{array}{c}{[n]} \\ k\end{array}\right)$ is a positroid if and only if

(1) Every edge of $P_{\mathcal{M}}$ has the form $\left[e_{I}, e_{J}\right]$, for $I, J \in\left(\begin{array}{c}{[n]} \\ k\end{array}\right)$ with $|I \cap J|=k-1$.

(2) Every facet of $P_{\mathcal{M}}$ is given by $x_{i}+x_{i+1}+\cdots+x_{j}=a_{i j}$ for some cyclic interval $\{i, i+1, \ldots, j\} \subset[n]$ and $a_{i j} \in \mathbb{Z}$.

Many of the results on the positive Grassmannian are based on an explicit birational parametrization [Pos06] of the positroid cells $\Pi_{\mathcal{M}}$ in terms of plabic graphs. In the next section we describe a more general class of Grassmannian graphs that includes plabic graphs.

\section{Grassmannian graphS}

Definition 4.1. A Grassmannian graph is a finite graph $G=(V, E)$, with vertex set $V$ and edge set $E$, embedded into a disk (and considered up to homeomorphism) with $n$ boundary vertices $b_{1}, \ldots, b_{n} \in V$ of degree 1 on the boundary of the disk (in the clockwise order), and possibly some internal vertices $v$ in the interior of the 
disk equipped with integer parameters $h(v) \in\{0,1, \ldots, \operatorname{deg}(v)\}$, called helicities of vertices. Here $\operatorname{deg}(v)$ is the degree of vertex $v$. We say that an internal vertex $v$ is of type $(h, d)$ if $d=\operatorname{deg}(v)$ and $h=h(v)$.

The set of internal vertices of $G$ is denoted by $V_{\text {int }}=V \backslash\left\{b_{1}, \ldots, b_{n}\right\}$, and the set of internal edges, i.e., the edges which are not adjacent to the boundary vertices, is denoted by $E_{\text {int }} \subset E$. The internal subgraph is $G_{\text {int }}=\left(V_{\text {int }}, E_{\text {int }}\right)$.

A perfect orientation of a Grassmannian graph $G$ is a choice of directions for all edges $e \in E$ of the graph $G$ such that, for each internal vertex $v \in V_{\text {int }}$ with helicity $h(v)$, exactly $h(v)$ of the edges adjacent to $v$ are directed towards $v$ and the remaining $\operatorname{deg}(v)-h(v)$ of adjacent edges are directed away from $v$. A Grassmannian graph is called perfectly orientable if it has a perfect orientation.

The helicity of a Grassmannian graph $G$ with $n$ boundary vertices is the number $h(G)$ given by

$$
h(G)-n / 2=\sum_{v \in V_{\text {int }}}(h(v)-\operatorname{deg}(v) / 2) .
$$

For a perfect orientation $\mathcal{O}$ of $G$, let $I(\mathcal{O})$ be the set of indices $i \in[n]$ such that the boundary edge adjacent to $b_{i}$ is directed towards the interior of $G$ in the orientation $\mathcal{O}$.

Lemma 4.2. For a perfectly orientable Grassmannian graph $G$ and any perfect orientation $\mathcal{O}$ of $G$, we have $|I(\mathcal{O})|=h(G)$. In particular, in this case, $h(G) \in$ $\{0,1, \ldots, n\}$.

Remark 4.3. This lemma expresses the Helicity Conservation Law. We leave it as an exercise for the reader.

For a perfectly orientable Grassmannian graph $G$ of helicity $h(G)=k$, let

$$
\mathcal{M}(G)=\{I(\mathcal{O}) \mid \mathcal{O} \text { is a perfect orientation of } G\} \subset\left(\begin{array}{c}
{[n]} \\
k
\end{array}\right) .
$$

Here is one result that links Grassmannian graphs with positroids.

Theorem 4.4. For a perfectly orientable Grassmannian graph $G$ with $h(G)=k$, the set $\mathcal{M}(G)$ is a positroid of rank $k$. All positroids have form $\mathcal{M}(G)$ for some $G$.

Definition 4.5. A strand $\alpha$ in a Grassmannian graph $G$ is a directed walk along edges of $G$ that either starts and ends at some boundary vertices, or is a closed walk in the internal subgraph $G_{\text {int }}$, satisfying the following Rules of the Road: For each internal vertex $v \in V_{\text {int }}$ with adjacent edges labelled $a_{1}, \ldots, a_{d}$ in the clockwise order, where $d=\operatorname{deg}(v)$, if $\alpha$ enters $v$ through the edge $a_{i}$, it leaves $v$ through the edge $a_{j}$, where $j=i+h(v)(\bmod d)$.

A Grassmannian graph $G$ is reduced if

(1) There are no strands which are closed loops in the internal subgraph $G_{\text {int }}$.

(2) All strands in $G$ are simple curves without self-intersections. The only exception is that we allow strands $b_{i} \rightarrow v \rightarrow b_{i}$ where $v \in V_{\text {int }}$ is a boundary leaf, that is a vertex of degree 1 connected with $b_{i}$ by an edge.

(3) Any two strands $\alpha \neq \beta$ cannot have a bad double crossing, that is, a pair of vertices $u \neq v$ such that both $\alpha$ and $\beta$ pass through $u$ and $v$ and both are directed from $u$ to $v$. (We allow double crossings where $\alpha$ goes from $u$ to $v$ and $\beta$ goes from $v$ to $u$.) 
(4) The graph $G$ has no vertices of degree 2 .

The decorated strand permutation $w=w_{G}$ of a reduced Grassmannian graph $G$ is the permutation $w:[n] \rightarrow[n]$ with fixed points colored in colors 0 or 1 such that

(1) $w(i)=j$ if the strand that starts at the boundary vertex $b_{i}$ ends at the boundary vertex $b_{j}$.

(2) For a boundary leaf $v$ connected to $b_{i}$, the decorated permutation $w$ has fixed point $w(i)=i$ colored in color $h(v) \in\{0,1\}$.

A complete reduced Grassmannian graph $G$ of type $(k, n)$, for $0 \leq k \leq n$, is a reduced Grassmannian graph whose decorated strand permutation is given by $w(i)=i+k(\bmod n)$. In addition, for $k=0$ (resp., for $k=n)$, we require that $G$ only has $n$ boundary leaves of helicity 0 (resp., of helicity 1) and no other internal vertices.

Theorem 4.6. $c f$. Pos06, Corollaries 14.7 and 14.10] (1) For any permutation $w:[n] \rightarrow[n]$ with fixed points colored in 0 or 1 , there exists a reduced Grassmannian graph $G$ whose decorated strand permutation $w_{G}$ is $w$.

(2) Any reduced Grassmannian graph is perfectly orientable. Moreover, it has an acyclic perfect orientation.

(3) A reduced Grassmannian graph $G$ is complete of type $(k, n)$ if and only if its helicity equals $h(G)=k$ and the number of internal faces (excluding $n$ boundary faces) equals

$$
f(k, n)-\sum_{v \in V_{\mathrm{int}}} f(h(v), \operatorname{deg}(v)) .
$$

where $f(k, n)=(k-1)(n-k-1)$. A reduced Grassmannian graph is complete if and only if it is not a proper induced subgraph of a larger reduced Grassmannian graph.
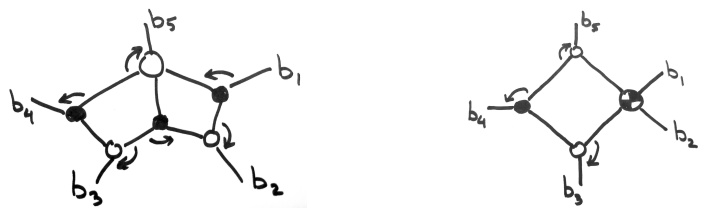

Figure 1. Two complete reduced Grassmannian graphs of type $(2,5)$ with 2 internal faces (left) and 1 internal face (right). The internal vertices of types $(1,3)$ and $(1,4)$ are colored in white, the type $(2,3)$ vertices colored in black, and the type $(2,4)$ vertex is "chessboard" colored.

Let us now describe a partial ordering and an equivalence relation on Grassmannian graphs.

Definition 4.7. For two Grassmannian graphs $G$ and $G^{\prime}$, we say that $G$ refines $G^{\prime}$ (and that $G^{\prime}$ coarsens $G$ ), if $G$ can be obtained from $G^{\prime}$ by a sequence of the following operations: Replace an internal vertex of type $(h, d)$ by a complete reduced Grassmannian graph of type $(h, d)$.

The refinement order on Grassmannian graphs is the partial order $G \leq_{\mathrm{ref}} G^{\prime}$ if $G$ refines $G^{\prime}$. We say that $G^{\prime}$ covers $G$, if $G^{\prime}$ covers $G$ in the refinement order. 
Two Grassmannian graphs $G$ and $G^{\prime}$ are refinement-equivalent if they are in the same connected component of the refinement order $\leq_{\text {ref }}$, that is, they can be obtained from each other by a sequence of refinements and coarsenings.

Definition 4.8. A Grassmannian graph is called a plabic graph if it is a minimal element in the refinement order.

The following is clear.

Lemma 4.9. A Grassmannian graph is a plabic graph if and only if each internal vertex in the graph has type $(1,3),(2,3),(0,1)$, or $(1,1)$.

In drawings of plabic and Grassmannian graphs, we color vertices of types $(1, d)$ in white color, and vertices of types $(d-1, d)$ in black color.

Let us now describe almost minimal elements in the refinement order.

Definition 4.10. A Grassmannian graph $G$ is called almost plabic if it covers a plabic graph (a minimal element) in the refinement order.

For example, the two graphs shown on Figure 4 are almost plabic. The following lemma is also straightforward from the definitions.

Lemma 4.11. Each almost plabic Grassmannian graph $G$ has exactly one internal vertex (special vertex) of type $(1,4),(2,4),(3,4),(0,2),(1,2)$, or $(2,2)$, and all other internal vertices of types $(1,3),(2,3),(0,1)$, or $(1,1)$. An almost plabic graph with a special vertex of type of type $(1,4),(2,4)$, or $(3,4)$ covers exactly two plabic graphs. An almost plabic graph with a special vertex of type $(0,2),(1,2)$, or $(2,2)$ covers exactly one plabic graph.

Note that a reduced Grassmannian graph cannot contain any vertices of degree 2. So each reduced almost plabic graph covers exactly two reduced plabic graphs.

Definition 4.12. Two plabic graphs are connected by a move of type $(1,4),(2,4)$, or $(3,4)$, if they are both covered by an almost plabic graph with a special vertex of the corresponding type. Two plabic graphs $G$ and $G^{\prime}$ are move-equivalent if they can be obtained from each other by a sequence of such moves.
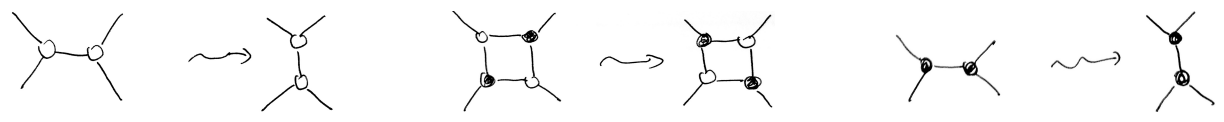

Figure 2. Three types of moves of plabic graphs: $(1,4)$ contraction-uncontraction of white vertices, $(2,4)$ square move, $(3,4)$ contraction-uncontraction of black vertices.

Let us say that vertices of types $(1,2),(0, d),(d, d)$ (except boundary leaves) are extraneous. A reduced graph cannot have a vertex of this form.

Theorem 4.13. (1) For two reduced Grassmannian graphs $G$ and $G^{\prime}$, the graphs are refinement-equivalent if and only if they have the same decorated strand permutation $w_{G}=w_{G^{\prime}}$.

(2) cf. Pos06, Theorem 13.4] For two reduced plabic graphs $G$ and $G^{\prime}$, the following are equivalent:

(a) The graphs are move-equivalent. 
(b) The graphs are refinement-equivalent.

(c) The graphs have the same decorated strand permutation $w_{G}=w_{G^{\prime}}$.

(3) A Grassmannian graph is reduced if and only if it has no extraneous vertices and is not refinement-equivalent to a graph with a pair of parallel edges (two edges between the same vertices), or a loop-edge (an edge with both ends attached to the same vertex).

(4) A plabic graph is reduced if and only if it has no extraneous vertices and is not move-equivalent to a plabic graph with a pair of parallel edges or a loop-edge.

Remark 4.14. Plabic graphs are similar to wiring diagrams that represent decompositions of permutations into products of adjacent transpositions. In fact, plabic graphs extend the notions of wiring diagrams and, more generally, double wiring diagrams of Fomin-Zelevinsky [FZ99, see [Pos06, Remark 14.8, Figure 18.1]. Moves of plabic graphs are analogous to Coxeter moves of decompositions of permutations. Reduced plabic graphs extend the notion of reduced decompositions of permutations.

Let us now summarize the results about the relationship between positroids, Grassmannian and plabic graphs, decorated permutations, and Grassmann necklaces. For a decorated permutation $w:[n] \rightarrow[n]$ (a permutation with fixed points colored 0 or 1$)$, define $\mathcal{J}(w):=\left(J_{1}, \ldots, J_{n}\right)$, where

$$
J_{i}=\left\{j \in[n] \mid c^{-i+1} w^{-1}(j)>c^{-i+1}(j)\right\} \cup\{j \in[n] \mid w(j)=j \text { colored } 1\} .
$$

The helicity of $w$ is defined as $h(w):=\left|J_{1}\right|=\cdots=\left|J_{n}\right|$. Conversely, for a Grassmann necklace $\mathcal{J}=\left(J_{1}, \ldots, J_{n}\right)$, let

$$
w(\mathcal{J}):=w, \quad \text { where } w(i)=\left\{\begin{array}{l}
j \text { if } J_{i+1}=\left(J_{i} \backslash\{i\}\right) \cup\{j\}, \\
i\left(\text { colored 0) if } i \notin J_{i}=J_{i+1},\right. \\
i\left(\text { colored 1) if } i \in J_{i}=J_{i+1} .\right.
\end{array}\right.
$$

Theorem 4.15. cf. Pos06 The following sets are in one-to-one correspondence:

(1) Positroids $\mathcal{M}$ of rank $k$ on $n$ elements.

(2) Decorated permutation $w$ of size $n$ and helicity $k$.

(3) Grassmann necklaces $\mathcal{J}$ of type $(k, n)$.

(4) Move-equivalence classes of reduced plabic graphs $G$ with $n$ boundary vertices and helicity $h(G)=k$.

(5) Refinement-equivalence classes of reduced Grassmannian graphs $G^{\prime}$ with $n$ boundary vertices and helicity $h\left(G^{\prime}\right)=k$.

The following maps (described above in the paper) give explicit bijection between these sets and form a commutative diagram:

(1) Reduced Grassmannian/plabic graphs to positroids: $G \mapsto \mathcal{M}(G)$.

(2) Reduced Grassmannian/plabic graphs to decorated permutations: $G \mapsto w_{G}$.

(3) Positroids to Grassmann necklaces: $\mathcal{M} \mapsto \mathcal{J}(\mathcal{M})$.

(4) Grassmann necklaces to positroids: $\mathcal{J} \mapsto \mathcal{M}(\mathcal{J})$,

(5) Grassmann necklaces to decorated permutations: $\mathcal{J} \mapsto w(\mathcal{J})$.

(6) Decorated permutations to Grassmann necklaces: $w \mapsto \mathcal{J}(w)$.

Proof of Theorems 4.4, 4.6, 4.13, 4.15. In case of plabic graphs, most of these results were proved in Pos06]. The extension of results to Grassmannian graphs follows from a few easy observations. 
Let $G$ and $G^{\prime}$ be a pair of Grassmannian graphs such that $G$ refines $G^{\prime}$. Any perfect orientation of $G$ induces a perfect orientation of $G^{\prime}$. Conversely, any perfect orientation of $G^{\prime}$ can be extended (not uniquely, in general) to a perfect orientation of $G$. Thus $G$ is perfectly orientable if and only if $G^{\prime}$ is perfectly orientable, and $\mathcal{M}(G)=\mathcal{M}\left(G^{\prime}\right)$ and $h(G)=h\left(G^{\prime}\right)$. Any strand of $G$ corresponds to a strand of $G^{\prime}$. The graph $G$ is reduced if and only if $G^{\prime}$ is reduced. If they are reduced, then they have the same decorated strand permutation $w_{G}=w_{G^{\prime}}$. Finally, any Grassmannian graph can be refined to a plabic graph. So the results for plabic graphs imply the results for Grassmannian graphs.

\section{WeAKLY SEPARATED COLLECTIONS AND CLUSTER ALGEBRAS}

Definition 5.1. [Sco05], cf. [LZ98] Two subsets $I, J \in\left(\begin{array}{c}{[n]} \\ k\end{array}\right)$ are weakly separated if there is no $a<b<c<d$ such that $a, c \in I \backslash J$ and $b, d \in J \backslash I$, or vise versa. A collection of subsets $S \subset\left(\begin{array}{c}{[n]} \\ k\end{array}\right)$ is weakly separated if it is pairwise weakly separated.

This is a variation of Leclerc-Zelevinsky's notion of weak separation LZ98 given by Scott Sco05. It appeared in their study of quasi-commuting quantum minors.

Definition 5.2. The face labelling of a reduced Grassmannian graph $G$ is the labelling of faces $F$ of $G$ by subsets $I_{F} \subset[n]$ given by the condition: For each strand $\alpha$ that goes from $b_{i}$ to $b_{j}$, we have $j \in I_{F}$ if and only if the face $F$ lies to the left of the strand $\alpha$ (with respect to the direction of the strand from $b_{i}$ to $b_{j}$ ).

Let us stay that two reduced plabic graphs are contraction-equivalent if they can be transformed to each other by the moves of type $(1,4)$ and $(3,4)$ (contractionuncontraction moves) without using the move of type $(2,4)$ (square move).

Theorem 5.3. OPS15] (1) Face labels of a reduced Grassmannian graph form a weakly separated collection in $\left(\begin{array}{c}{[n]} \\ k\end{array}\right)$, where $k=h(G)$ is the helicity of $G$.

(2) Every maximal by inclusion weakly separated collection in $\left(\begin{array}{c}{[n]} \\ k\end{array}\right)$ is the collection of face labels of a complete reduced plabic graph of type $(k, n)$.

(3) This gives a bijection between maximal by inclusion weakly separated collections in $\left(\begin{array}{c}{[n]} \\ k\end{array}\right)$, and contraction-equivalence classes of complete reduced plabic graphs of type $(k, n)$.

Remark 5.4. Weakly separated collections are related to the cluster algebra structure [FZ02a, FZ03, BFZ05, FZ07] on the Grassmannian studied by Scott Sco06. In general, the cluster algebra on $\operatorname{Gr}(k, n)$ has infinitely many clusters. (See [Sco06] for a classification of finite cases.) There is, however, a nicely behaved finite set of clusters, called the Plücker clusters, which are formed by subsets of the Plücker coordinates $\Delta_{I}$. According to OPS15, Theorem 1.6], the Plücker clusters for $\operatorname{Gr}(k, n)$ are exactly the sets $\left\{\Delta_{I}\right\}_{I \in S}$ associated with maximal weakly-separated collections $S \subset\left(\begin{array}{c}{[n]} \\ k\end{array}\right)$. They are in bijection with contraction-equivalence classes of type $(k, n)$ complete reduced plabic graphs, and are given by the $k(n-k)+1$ face labels of such graphs. Square moves of plabic graphs correspond to mutations of Plücker clusters in the cluster algebra.

Theorem 5.3 implies an affirmative answer to the purity conjecture of Leclerc and Zelevinsky [LZ98. An independent solution of the purity conjecture was given by Danilov, Karzanov, and Koshevoy [DKK10] in terms of generalized tilings. The relationship between the parametrization a positroid cell given by a plabic graph $G$ (see 
Section 7 below) and the Plücker cluster $\{\Delta\}_{I \in S}$ associated with the same graph $G$ induces a nontrivial transformation, called the twist map, which was explicitly described by Muller and Speyer MuS16. Weakly separated collections appeared in the study of arrangements of equal minors [FP16. In [GP17] the notion of weakly separated collections was extended in the general framework of oriented matroids and zonotopal tilings.

\section{Cyclically labelled Grassmannian}

Let us reformulate the definition of the Grassmannian and its positive part in a more invariant form, which makes its cyclic symmetry manifest. In the next section, we will consider "little positive Grassmannians" associated with vertices $v$ of a Grassmannian graph $G$ whose ground sets correspond to the edges adjacent to $v$. There is no natural total ordering on such a set of edges, however there is the natural cyclic (clockwise) ordering.

We say that a cyclic ordering of a finite set $C$ is a choice of closed directed cycle that visits each element of $C$ exactly once. A total ordering of $C$ is compatible with a cyclic ordering if it corresponds to a directed path on $C$ obtained by removing an edge of the cycle. Clearly, there are $|C|$ such total orderings.

Definition 6.1. Let $C$ be a finite set of indices with a cyclic ordering of its elements, and let $k$ be an integer between 0 and $|C|$. The cyclically labelled Grassmannian $\operatorname{Gr}(k, C)$ over $\mathbb{R}$ is defined as the subvariety of the projective space $\mathbb{P}\left(\begin{array}{c}|c| \\ k\end{array}\right)-1$ with projective Plücker coordinates $\left(\Delta_{I}\right)$ labelled by unordered $k$-element subsets $I \subset C$ satisfying the Plücker relations written with respect to any total order " $<$ " on $C$ compatible with the given cyclic ordering:

$$
\sum_{i \in A \backslash B}(-1)^{|\{a \in A, a>i\}|+|\{b \in B, b<i\}|} \Delta_{A \backslash\{i\}} \Delta_{B \cup\{i\}}=0,
$$

where $A$ and $B$ are any $(k+1)$-element and $(k-1)$-element subsets of $C$, respectively. (More precisely, $\operatorname{Gr}(k, C)$ is the projective algebraic variety given by the radical of the ideal generated by the above Plücker relations.)

The positive part $\operatorname{Gr}^{>0}(k, C)$ is the subset of $G r(k, C)$ where the Plücker coordinates can be simultaneously rescaled so that $\Delta_{I}>0$, for all $k$-element subsets $I \subset C$.

Remark 6.2. The Plücker relations (written as above) are invariant with respect to cyclic shifts of the ordering " $<$ ". Thus the definition of the cyclically labelled Grassmannian $\operatorname{Gr}(k, C)$ is independent of a choice of the total order on $C$. For example, for $k=2$ and $C=\{1,2,3,4\}, \operatorname{Gr}(2, C)$ is the subvariety of $\mathbb{P}^{6-1}$ given by the Plücker relation:

$$
\Delta_{\{1,3\}} \Delta_{\{2,4\}}=\Delta_{\{1,2\}} \Delta_{\{3,4\}}+\Delta_{\{1,4\}} \Delta_{\{2,3\}} .
$$

Observe the cyclic symmetry of this relation! The ordering of indices $2<3<4<1$ gives exactly the same $\operatorname{Gr}(2, C)$ with the same positive part $G r^{>0}(2, C)$.

Remark 6.3. There is a subtle yet important difference between the cyclically labelled Grassmannian $G r(k, C)$ with the Plücker coorinates $\Delta_{I}$ and the usual definition of the Grassmannian $G r(k, n), n=|C|$, with the "usual Plücker coordinates" defined as the minors $D_{\left(i_{1}, \ldots, i_{k}\right)}=\operatorname{det}\left(A_{i_{1}, \ldots, i_{k}}\right)$ of submatrices $A_{i_{1}, \ldots, i_{k}}$ of a $k \times n$ matrix $A$. 
The $D_{\left(i_{1}, \ldots, i_{k}\right)}$ are labelled by ordered collections $\left(i_{1}, \ldots, i_{k}\right)$ of indices. They are anti-symmetric with respect to permutations of the indices $i_{1}, \ldots, i_{k}$. On the other hand, the $\Delta_{\left\{i_{1}, \ldots, i_{k}\right\}}$ are labelled by unordered subsets $I=\left\{i_{1}, \ldots, i_{k}\right\}$. So they are symmetric with respect to permutations of the indices $i_{1}, \ldots, i_{k}$.

The "usual Plücker relations" for the $D_{\left(i_{1}, \ldots, i_{k}\right)}$ have the $S_{n}$-symmetry with respect to all permutations of the ground set. On the other hand, the above Plücker relations for the $\Delta_{\left\{i_{1}, \ldots, i_{k}\right\}}$ have only the $\mathbb{Z} / n \mathbb{Z}$-symmetry with respect to cyclic shifts of the ground set.

Of course, if we fix a total order of the ground set, we can rearrange the indices in $D_{\left(i_{1}, \ldots, i_{k}\right)}$ in the increasing order and identify $D_{\left(i_{1}, \ldots, i_{k}\right)}$, for $i_{1}<\cdots<i_{k}$, with $\Delta_{\left\{i_{1}, \ldots, i_{k}\right\}}$. This identifies the cyclically labelled Grassmannian $\operatorname{Gr}(k, C)$ with the usual Grassmannian $G r(k, n)$. However, this isomorphism is not canonical because it depends on a choice of the total ordering of the index set. For even $k$, the isomorphism is not invariant under cyclic shifts of the index set.

\section{Perfect orientation parametrization of Positroid CELls}

Positroid cells were parametrized in Pos06 in terms of boundary measurements of perfect orientations of plabic graphs. Equivalent descriptions of this parametrization were given in terms of network flows by Talaska Tal08 and in terms of perfect matchings [PSW09, Lam15]. Another interpretation of this parametrization was motivated by physics ABCGPT16, where plabic graphs were viewed as on-shell diagrams, whose vertices represent little Grassmannians $\operatorname{Gr}(1,3)$ and $\operatorname{Gr}(2,3)$ and edges correspond to gluings, see also [Lam15, Section 14] for a more mathematical description. Here we give a simple and invariant way to describe the parametrization in the general setting of Grassmannian graphs and their perfect orientations. It easily specializes to all the other descriptions. Yet it clarifies the idea of gluings of little Grassmannians.

Let $G=(V, E)$ be a perfectly orientable Grassmannian graph with $n$ boundary vertices and helicity $h(G)=k$, and let $G_{\text {int }}=\left(V_{\text {int }}, E_{\text {int }}\right)$ be its internal subgraph. Also let $E_{\text {bnd }}=E \backslash E_{\text {int }}$ be the set of boundary edges of $G$.

Informally speaking, each internal vertex $v \in V_{\text {int }}$ represents the "little Grassmannian" $\operatorname{Gr}(h, d)$, where $d$ is the degree of vertex $v$ and $h$ is its helicity. We "glue" these little Grassmannians along the internal edges $e \in E_{\text {int }}$ of the graph $G$ to form a subvariety in the "big Grassmannian" $G r(k, n)$. Gluing along each edge kills one parameter. Let us give a more rigorous description of this construction.

For an internal vertex $v \in V_{\text {int }}$, let $E(v) \subset E$ be the set of all adjacent edges to $v$ (possibly including some boundary edges), which is cyclically ordered in the clockwise order (as we go around $v$ ). Define the positive vertex-Grassmannian $G r^{>0}(v)$ as the positive part of the cyclically labelled Grassmannian

$$
G r^{>0}(v):=G r^{>0}(h(v), E(v)) .
$$

Let $\left(\Delta_{J}^{(v)}\right)$ be the Plücker coordinates on $G r^{>0}(v)$, where $J$ ranges over the set $\left(\begin{array}{c}E(v) \\ h(v)\end{array}\right)$ of all $h(v)$-element subsets in $E(v)$.

Let us define several positive tori (i.e., positive parts of complex tori). The boundary positive torus is $T_{\text {bnd }}^{>0}:=\left(\mathbb{R}_{>0}\right)^{E_{\mathrm{bnd}}} \simeq\left(\mathbb{R}_{>0}\right)^{n}$. The internal positive torus is $T_{\mathrm{int}}^{>0}:=\left(\mathbb{R}_{>0}\right)^{E_{\mathrm{int}}}$, and the total positive torus $T_{\mathrm{tot}}^{>0}:=T_{\mathrm{bnd}}^{>0} \times T_{\mathrm{int}}^{>0}$. The boundary/internal/total positive torus is the group of $\mathbb{R}_{>0}$-valued functions on boundary/internal/all edges of $G$. 
These tori act on the positive vertex-Grassmannians $G r^{>0}(v)$ by rescaling the Plücker coordinates. For $\left(t_{e}\right)_{e \in E} \in T_{\text {tot }}^{>0}$,

$$
\left(t_{e}\right):\left(\Delta_{J}^{(v)}\right) \longmapsto\left(\left(\prod_{e \in J} t_{e}\right) \Delta_{J}^{(v)}\right) .
$$

The boundary torus $T_{\text {bnd }}^{>0}$ also acts of the "big Grassmannian" $G r(k, n)$ as usual $\left(t_{1}, \ldots, t_{n}\right): \Delta_{I} \mapsto\left(\prod_{i \in I} t_{i}\right) \Delta_{I}$, for $\left(t_{1}, \ldots, t_{n}\right) \in T_{\mathrm{bnd}}^{>0}$.

Recall that, for a perfect orientation $\mathcal{O}$ of $G, I(\mathcal{O})$ denotes the set of $i \in[n]$ such that the boundary edge adjacent to $b_{i}$ is directed towards the interior of $G$ in $\mathcal{O}$. For an internal vertex $v \in V_{\text {int }}$, let $J(v, \mathcal{O}) \subset E(v)$ be the subset of edges adjacent to $v$ which are directed towards $v$ in the orientation $\mathcal{O}$.

We are now ready to describe the perfect orientation parametrization of the positroid cells.

Theorem 7.1. Let $G$ be a perfectly orientable Grassmannian graph. Let $\mu_{G}$ be the map defined on the direct product of the positive vertex-Grassmannians $G r>0(v)$ and written in terms of the Plücker coordinates as

$$
\begin{aligned}
& \mu_{G}: \underset{v \in V_{\mathrm{int}}}{X} G r^{>0}(v) \longrightarrow \mathbb{P}^{\left(\begin{array}{c}
{[n]} \\
k
\end{array}\right)-1} \\
& \mu_{G}: \underset{v \in V_{\mathrm{int}}}{X}\left(\Delta_{J}^{(v)}\right)_{J \in\left(\begin{array}{c}
E(v) \\
h(v)
\end{array}\right)} \longmapsto\left(\Delta_{I}\right)_{I \in\left(\begin{array}{c}
{[n]} \\
k
\end{array}\right)},
\end{aligned}
$$

where $\Delta_{I}$ is given by the sum over all perfect orientations $\mathcal{O}$ of the graph $G$ such that $I(\mathcal{O})=I$ :

$$
\Delta_{I}=\sum_{I(\mathcal{O})=I} \prod_{v \in V_{\mathrm{int}}} \Delta_{J(v, \mathcal{O})}^{(v)}
$$

(1) The image of $\mu_{G}$ is exactly the positroid cell $\Pi_{\mathcal{M}} \subset G r^{\geq 0}(k, n) \subset \mathbb{P}^{\left[\begin{array}{c}{[n]} \\ k\end{array}\right)-1}$, where $\mathcal{M}=\mathcal{M}(G)$ is the positroid associated with $G$.

(2) The $\operatorname{map} \mu_{G}$ is $T_{\mathrm{int}}^{>0}$-invariant and $T_{\mathrm{bnd}}^{>0}$-equivariant, that is, $\mu_{G}(t \cdot x)=\mu_{G}(x)$ for $t \in T_{\text {int }}^{>0}$, and $\mu_{G}\left(t^{\prime} \cdot x\right)=t^{\prime} \cdot \mu_{G}(x)$ for $t^{\prime} \in T_{\text {bnd }}^{>0}$.

(3) The map $\mu_{G}$ induces the birational subtraction-free bijection $\bar{\mu}_{G}$

$$
\bar{\mu}_{G}:\left(\underset{v \in V_{\text {int }}}{\times} G r^{>0}(v)\right) / T_{\text {int }}^{>0} \longrightarrow \Pi_{G}
$$

if and only if the Grassmannian graph $G$ is a reduced.

Remark 7.2. The phrase "birational subtraction-free bijection" means that both $\bar{\mu}_{G}$ and its inverse $\left(\bar{\mu}_{G}\right)^{-1}$ can be expressed in terms of the Plücker coordinates by rational (or even polynomial) expressions written without using the "-" sign.

Proof. Part (2) is straightforward from the definitions. Let us first prove the remaining claims in the case when $G$ is a plabic graph. In fact, in this case this construction gives exactly the boundary measurement parametrization of $\Pi_{G}$ from [Pos06, Section 5]. The Plücker coordinates for the boundary measurement parametrization were given in [Pos06, Proposition 5.3] and expressed by Talaska Tal08, Theorem 1.1] in terms of network flows on the graph $G$. The construction of the boundary measurement parametrization (and Talaska's formula) depends on a choice of a reference perfect orientation $\mathcal{O}_{0}$. One observes that any other perfect 
orientation $\mathcal{O}$ of the plabic graph $G$ is obtained from $\mathcal{O}_{0}$ by reversing the edges along a network flow, which gives a bijection between network flows and perfect orientations of $G$. This shows that the above expression for $\Delta_{I}$ is equivalent to Talaska's formula, which proves the equivalence of the above perfect orientation parametrization and the boundary measurement parametrization from Pos06. Parts (1) and (3) now follow from results of [Pos06].

For an arbitrary Grassmannian graph $G^{\prime}$, let $G$ be a plabic graph that refines $G^{\prime}$. We already know that each "little plabic graph" $G_{v}$, i.e., the subgraph of $G$ that refines a vertex $v$ of $G^{\prime}$, parametrizes each positive vertex-Grassmannian $G r^{>0}(v)$ by a birational subtraction-free bijection $\bar{\mu}_{v}:=\bar{\mu}_{G_{v}}$. We also know the map $\bar{\mu}_{G}$ for the plabic graph $G$ parametrizes the cell $\Pi_{\mathcal{M}(G)}$ if $G$ reduced, or maps surjectively but not bijectively onto $\Pi_{\mathcal{M}(G)}$ if $G$ is not reduced. Then the map $\bar{\mu}_{G^{\prime}}$ is given by the composition $\bar{\mu}_{G} \circ\left(\times_{v \in V_{\text {int }}}\left(\bar{\mu}_{v}\right)^{-1}\right)$ and the needed result follows.

The above construction can be thought of as a gluing of the "big Grassmannian" out of "little Grassmannians." This is similar to a tiling of a big geometric object (polytope) by smaller pieces (smaller polytopes). As we will see, this construction literally corresponds to certain subdivisions of polytopes.

\section{Polyhedral subdivisions: Baues poset And fiBer Polytopes}

In this section we discuss Billera-Sturmfels' theory [BS92] of fiber polytopes, the generalized Baues problem [BKS94, and flip-connectivity, see also [Rei99, RS00, ARS99, Ath01, AS02 for more details.

8.1. The Baues poset of $\pi$-induced subdivisions. Let $\pi: P \rightarrow Q$ be an affine projection from one convex polytope $P$ to another convex polytope $Q=\pi(P)$.

Informally, a $\pi$-induced polyhedral subdivision is a collection of faces of the polytope $P$ that projects to a polyhedral subdivision of the polytope $Q$.

Here is a rigorous definition, see BS92. Let $A$ be the multiset of projections $\pi(v)$ of vertices $v$ of $P$. Each element $\pi(v)$ of $A$ is labelled by the vertex $v$. For $\sigma \subset A$, let $\operatorname{conv}(\sigma)$ denotes the convex hull of $\sigma$. We say that $\sigma^{\prime} \subset \sigma$ is a face of $\sigma$ if $\sigma^{\prime}$ consists of all elements of $\sigma$ that belong to a face of the polytope $\operatorname{conv}(\sigma)$.

A $\pi$-induced subdivision is a finite collection $S$ of subsets $\sigma \subset A$, called cells, such that

(1) Each $\sigma \in S$ is the projection under $\pi$ of the vertex set of a face of $P$.

(2) For each $\sigma \in S$, $\operatorname{dim}(\operatorname{conv}(\sigma))=\operatorname{dim}(Q)$.

(3) For any $\sigma_{1}, \sigma_{2} \in S, \operatorname{conv}\left(\sigma_{1}\right) \cap \operatorname{conv}\left(\sigma_{2}\right)=\operatorname{conv}\left(\sigma_{1} \cap \sigma_{2}\right)$.

(4) For any $\sigma_{1}, \sigma_{2} \in S, \sigma_{1} \cap \sigma_{2}$ is either empty or a face of both $\sigma_{1}$ and $\sigma_{2}$.

(5) $\bigcup_{\sigma \in S} \operatorname{conv}(\sigma)=Q$.

The Baues poset $\omega(P \stackrel{\pi}{\rightarrow} Q)$ is the poset of all $\pi$-induced subdivisions partially ordered by refinement, namely, $S \leq T$ means that, for every cell $\sigma \in S$, there exists a cell $\tau \in T$ such that $\sigma \subset \tau$. This poset has a unique maximal element $\hat{1}$, called the trivial subdivision, that consists of a single cell $\sigma=A$. All other elements are called proper subdivisions. Let $\hat{\omega}(P \stackrel{\pi}{\rightarrow} Q)):=\omega(P \stackrel{\pi}{\rightarrow} Q)-\hat{1}$ be the poset of proper $\pi$-induced subdivisions obtained by removing the maximal element $\hat{1}$. The minimal elements of the Baues poset are called tight $\pi$-induced subdivisions.

Among all $\pi$-induced subdivisons, there is a subset of coherent subdivisions that come from linear height functions $h: P \rightarrow \mathbb{R}$ as follows. For each $q \in Q$, let 
$\bar{F}_{q}$ be the face of the fiber $\pi^{-1}(q) \cap P$ where the height function $h$ reaches its maximal value. The face $\bar{F}_{q}$ lies in the relative interior of some face $F_{q}$ of $P$. The collection of faces $\left\{F_{q}\right\}_{q \in Q}$ projects to a $\pi$-induced subdivision of $Q$. Let $\omega_{\text {coh }}(P \stackrel{\pi}{\rightarrow}$ $Q) \subseteq \omega(P \stackrel{\pi}{\rightarrow} Q)$ be the subposet of the Baues poset formed by the coherent $\pi$ induced subdivisions. This coherent part of the Baues poset is isomorphic to the face lattice of the convex polytope $\Sigma(P \stackrel{\pi}{\rightarrow} Q)$, called the fiber polytope, defined as the Minkowskii integral of fibers of $\pi$ (the limit Minkowskii sums):

$$
\Sigma(P \stackrel{\pi}{\rightarrow} Q):=\int_{q \in Q}\left(\pi^{-1}(q) \cap P\right) d q
$$

In general, the whole Baues poset $\omega(P \stackrel{\pi}{\rightarrow} Q)$ may not be polytopal.

8.2. The generalized Baues problem and flip-connectivity. For a finite poset $\omega$, the order complex $\Delta \omega$ is the simplicial complex of all chains in $\omega$. The "topology of a poset $\omega$ " means the topology of the simplicial complex $\Delta \omega$. For example, if $\omega$ is the face poset of a regular cell complex $\Delta$, then $\Delta \omega$ is the barycentric subdivision of the cell complex $\Delta$; and, in particular, $\Delta \omega$ is homeomorphic to $\Delta$.

Clearly, the subposet $\hat{\omega}_{\text {coh }}(P \stackrel{\pi}{\rightarrow} Q)$ of proper coherent $\pi$-induced subdivisions homotopy equivalent to a $(\operatorname{dim}(P)-\operatorname{dim}(Q)-1)$-sphere, because it is the face lattice of a convex polytope of $\operatorname{dimension} \operatorname{dim}(P)-\operatorname{dim}(Q)$, namely, the fiber polytope $\Sigma(P \stackrel{\pi}{\rightarrow} Q)$.

The generalized Baues problem (GBP) posed by Billera, Kapranov, Sturmfels BKS94 asks whether the same is true about the poset of all proper $\pi$-induced subdivisions. Is it true that $\hat{\omega}(P \stackrel{\pi}{\rightarrow} Q)$ is homotopy equivalent to a $(\operatorname{dim}(P)-$ $\operatorname{dim}(Q)-1)$-sphere? In general, the GBP is a hard question. Examples of Baues posets with disconnected topology were constructed by Rambau and Ziegler RZ96. and more recently by Liu Liu16. There are, however, several general classes of projections of polytopes, where the GBP has an affirmative answer, see the next section.

Another related question is about connectivity by flips. For a projection $\pi$ : $P \rightarrow Q$, the flip graph is the restriction of the Hasse diagram of the Baues poset $\omega(P \stackrel{\pi}{\rightarrow} Q)$ to elements of rank 0 (tight subdivisions) and rank 1 (subdivisions that cover a tight subdivision). The elements of rank 1 in the flip graph are called flips. The flip-connectivity problem asks whether the flip graph is connected. The coherent part of the flip graph is obviously connected, because it is the 1-skeleton of the fiber polytope $\Sigma(P \stackrel{\pi}{\rightarrow} Q)$.

The GBP and the flip-connectivity problem are related to each other, but, strictly speaking, neither of them implies the other, see [Rei99, Section 3] for more details.

8.3. Triangulations and zonotopal tilings. There are two cases of the above general setting that attracted a special attention in the literature.

The first case is when the polytope $P$ is the $(n-1)$-dimensional simplex $\Delta^{n-1}$. The multiset $A$ of projections of vertices of the simplex can be an arbitrary multiset of $n$ points, and $Q=\operatorname{conv}(A)$ can be an arbitrary convex polytope. In this case, the Baues poset $\omega\left(\Delta^{n-1} \stackrel{\pi}{\rightarrow} Q\right)$ is the poset of all polyhedral subdivisions of $Q$ (with vertices at $A$ ); tight $\pi$-induced subdivisions are triangulations of $Q$; and the fiber polytope $\Sigma\left(\Delta^{n-1} \stackrel{\pi}{\rightarrow} Q\right)$ is exactly is the secondary polytope of Gelfand-KapranovZelevinsky [GKZ94, which appeared in the study of discriminants. 
In particular, for a projection of the simplex $\Delta^{n-1}$ to an $n$-gon $Q, \pi$-induced subdivisions are exactly the subdivisions of the $n$-gon by noncrossing chords. All of them are coherent. Tight subdivisions are triangulations of the $n$-gon. There are the Catalan number $C_{n-2}=\frac{1}{n-1}\left(\begin{array}{c}2 n-4 \\ n-2\end{array}\right)$ of triangulations of the $n$-gon. The fiber polytope (or the secondary polytope) in this case is the Stasheff associahedron.

Another special case is related to projections $\pi: P \rightarrow Q$ of the hypercube $P=$ 四 $n:=[0,1]^{n}$. The projections $Q=\pi\left(\mathbb{U}_{n}\right)$ of the hypercube form a special class of polytopes, called zonotopes. In this case, $\pi$-induced subdivisions are zonotopal tilings of zonotopes $Q$. According to Bohne-Dress theorem Boh92, zonotopal tilings of $Q$ are in bijection with 1-element extensions of the oriented matroid associated with the zonotope $Q$.

For a projection of the $n$-hypercube 四 $n$ to a 1 -dimensional line segment, the fiber polytope is the permutohedron. For a projection $\pi: \mathbb{U}_{n} \rightarrow Q$ of the $n$-hypercube 四 $n$ to a $2 n$-gon $Q$, fine zonotopal tilings (i.e., tight $\pi$-induced subdivisions) are known as rhomus tilings of the $2 n$-gon. They correspond to commutation classes of reduced decompositions of the longest permutation $w_{\circ} \in S_{n}$.

\section{CyCliC POLytopes AND CyClic ZONOTOPES}

Fix two integers $n$ and $0 \leq d \leq n-1$.

Definition 9.1. A cyclic projection is a linear map

$$
\pi: \mathbb{R}^{n} \rightarrow \mathbb{R}^{d+1}, \quad \pi: x \mapsto M x
$$

given by a $(d+1) \times n$ matrix $M=\left(u_{1}, \ldots, u_{n}\right)$ (the $u_{i}$ are the column vectors) with all positive maximal $(d+1) \times(d+1)$ minors and such that $f\left(u_{1}\right)=\cdots=f\left(u_{n}\right)=1$ for some linear form $f: \mathbb{R}^{d+1} \rightarrow \mathbb{R}$. In other words, $M$ represents a point of the positive Grassmannian $G r^{>0}(d+1, n)$ with columns $u_{i}$ rescaled so that they all lie on the same affine hyperplane $H_{1}=\left\{y \in \mathbb{R}^{d+1} \mid f(y)=1\right\}$.

The cyclic polytope is the image under a cyclic projection $\pi$ of the standard $(n-1)$-dimensional simplex $\Delta^{n-1}:=\operatorname{conv}\left(e_{1}, \ldots, e_{n}\right)$

$$
C(n, d):=\pi\left(\Delta^{n-1}\right) \subset H_{1} .
$$

The cyclic zonotope is the image of the standard $n$-hypercube $\mathbb{U}_{n}:=[0,1]^{n} \subset \mathbb{R}^{n}$

$$
Z(n, d+1):=\pi\left(\text { 四 }_{n}\right) \subset \mathbb{R}^{d+1} .
$$

Remark that, for each $n$ and $d$, there are many combinatorially (but not linearly) isomorphic cyclic polytopes $C(n, d)$ and cyclic zonotopes $Z(n, d+1)$ that depend on a choice of the cyclic projection $\pi$. Clearly, $C(n, d)=Z(n, d+1) \cap H_{1}$.

Ziegler Zie93] identified fine zonotopal tilings of the cyclic zonotope $Z(n, d+1)$, i.e., the minimal elements of the Baues poset $\omega\left(\mathbb{U}_{n} \stackrel{\pi}{\rightarrow} Z(n, d+1)\right)$, with elements of Manin-Shekhtman's higher Bruhat order [MaS86], also studied by Voevodsky and Kapranov [VK91]. According to results of Sturmfels and Ziegler [SZ93], Ziegler [Zie93], Rambau Ram97, and Rambau and Santos [RS00, the GBP and flipconnectivity have affirmative answers in these cases.

Theorem 9.2. (1) SZ93] For $\pi:$ 四 $n \rightarrow Z(n, d+1)$, the poset of proper zonotopal tilings of the cyclic zonotope $Z(n, d+1)$ is homotopy equivalent to an $(n-d-2)$ dimensional sphere. The set of fine zonotopal tilings of $Z(n, d+1)$ is connected by flips. 
(2) RS00 For $\pi: \Delta^{n-1} \rightarrow C(n, d)$, the poset of proper subdivisions of the cyclic polytope $C(n, d)$ is homotopy equivalent to an $(n-d-2)$-dimensional sphere. Ram97. The set of triangulations of the cyclic polytope $C(n, d)$ is connected by flips.

\section{CyClic PRojections of The hypersimpleX}

Fix three integers $0 \leq k \leq n$ and $0 \leq d \leq n-1$. The hypersimplex $\Delta_{k n}:=$ conv $\left\{e_{I} \mid I \in\left(\begin{array}{c}{[n]} \\ k\end{array}\right)\right\}$ is the $k$-th section of the $n$-hypercube ${ }_{n} \subset \mathbb{R}^{n}$

$$
\Delta_{k n}=\text { 团 }_{n} \cap\left\{x_{1}+\cdots+x_{n}=k\right\} .
$$

Let $\pi: \mathbb{R}^{n} \rightarrow \mathbb{R}^{d+1}$ be a cyclic projection as above. Define the polytope

$$
Q(k, n, d):=\pi\left(\Delta_{k n}\right)=Z(n, d+1) \cap H_{k},
$$

where $H_{k}$ is the affine hyperplane $H_{k}:=\left\{y \in \mathbb{R}^{d+1} \mid f(y)=k\right\}$. Clearly, for $k=1$, the polytope $Q(1, n, d)$ is the cyclic polytope $C(n, d)$.

Let $\omega(k, n, d)$ be the Baues poset of $\pi$-induced subdivisions for a cyclic projection $\pi: \Delta_{k n} \rightarrow Q(k, n, d):$

$$
\omega(k, n, d):=\omega\left(\Delta_{k n} \stackrel{\pi}{\rightarrow} Q(k, n, d)\right) .
$$

Let $\omega_{\text {coh }}^{\pi}(k, n, d):=\omega_{\text {coh }}\left(\Delta_{k n} \stackrel{\pi}{\rightarrow} Q(k, n, d)\right) \subseteq \omega(k, n, d)$ be its coherent part. Note that the coherent part $\omega_{\mathrm{coh}}^{\pi}(k, n, d)$ depends on a choice of the cyclic projection $\pi$, but the whole poset $\omega(k, n, d)$ is independent of any choices. The coherent part $\omega_{\text {coh }}^{\pi}(k, n, d)$ may not be equal $\omega(k, n, d)$. For example they are not equal for $(k, n, d)=(3,6,2)$.

The poset $\omega(k, n, d)$ is a generalization of the Baues poset of subdivisions of the cyclic polytope $C(n, d)$, and is related to the Baues poset of zonotopal tilings of the cyclic zonotope $Z(n, d+1)$ in an obvious manner. For $k=1, \omega(1, n, d)=$ $\omega\left(\Delta^{n-1} \stackrel{\pi}{\rightarrow} C(n, d)\right)$. For any $k$, there is the order preserving $k$-th section map

$$
\operatorname{Section}_{k}: \omega\left(\text { 四 }{ }_{n} \stackrel{\pi}{\rightarrow} Z(n, d+1)\right) \rightarrow \omega(k, n, d)
$$

that send a zonotopal tiling of $Z(n, d+1)$ to its section by the hyperplane $H_{k}$.

Let $\omega_{\text {lift }}(k, n, d) \subseteq \omega(k, n, d)$ be the image of the map $\operatorname{Section}_{k}$. We call the elements of $\omega_{\text {lift }}(k, n, d)$ the lifting $\pi$-induced subdivisions. They form the subset of $\pi$-induced subdivisions from $\omega(k, n, d)$ that can be lifted to a zonotopal tiling of the cyclic zonotope $Z(n, d+1)$. Clearly, we have

$$
\omega_{\text {coh }}^{\pi}(k, n, d) \subseteq \omega_{\text {lift }}(k, n, d) \subseteq \omega(k, n, d) .
$$

The equality of the sets of minimal elements of $\omega(k, n, d)$ and $\omega_{\text {lift }}(k, n, d)$ was proved in the case $k=1$ by Rambau and Santos [RS00, who showed that all triangulations of the cyclic polytope $C(n, d)$ are lifting triangulations. For $d=2$, the equality follows from the result of Galashin Gal16. (Theorem 11.7 below) about plabic graphs, as we will explain in the next section.

Theorem 10.1. The minimal elements of the posets $\omega_{\mathrm{lift}}(k, n, d)$ and $\omega(k, n, d)$ are the same in the following cases: (1) $k=1$ and any $n, d ;(2) d=2$ and any $k, n$.

Flip-connectivity [SZ93, Zie93] of zonotopal tilings of $Z(n, d+1)$ easily implies the following claim.

Lemma 10.2. The minimal elements of $\omega_{\text {lift }}(k, n, d)$ are connected by flips. 
Indeed, for any pair of fine zonotopal tilings $T$ and $T^{\prime}$ of $Z(n, d+1)$ connected by a flip, their $k$-sections $\operatorname{Section}_{k}(T)$ and $\operatorname{Section}_{k}\left(T^{\prime}\right)$ are either equal to each other or connected by a flip.

The Baues posets of the form $\omega(k, n, d)$ are good candidates for a general class of projections of polytopes where the GBP and flip-connectivity problem might have affirmative answers.

Problem 10.3. Is the poset $\omega(k, n, d)-\hat{1}$ homotopy equivalent of a sphere? Can its minimal elements be connected by flips? Is it true that $\omega_{\mathrm{lift}}(k, n, d)=\omega(k, n, d)$ ?

Example 10.4. For $d=1$, the Baues poset $\omega(k, n, 1)$ is already interesting. Its minimal elements correspond to monotone paths on the hypersimplex $\Delta_{k n}$, which are increasing paths that go along the edges of the hypersimplex $\Delta_{k n}$. Such paths are the subject of the original (non-generalized) Baues problem Bau80, which was proved by Billera, Kapranov, Sturmfels BKS94 (for any 1-dimensional projection of a polytope). More specifically, monotone paths on $\Delta_{k n}$ correspond to directed paths from $[1, k]$ to $[n-k+1, n]$ in the directed graph on $\left(\begin{array}{c}{[n]} \\ k\end{array}\right)$ with edges $I \rightarrow J$ if $J=(I \backslash\{i\}) \cup\{j\}$ for $i<j$.

It is not hard to see that, for $k=1, n-1$, there are $2^{n-2}$ monotone paths, and the posets $\omega(1, n, 1)$ and $\omega(n-1, n, 1)$ are isomporphic to the Boolean lattice $B_{n-2}$, i.e., the face poset of the hypercube $\mathbb{U}_{n-2}$. For $n=2,3,4,5$, the Baues poset $\omega(2, n, 1)$ has $1,2,10,62$ minimal elements.

Monotone paths on $\Delta_{k n}$ might have different lengths. The longest monotone paths are in an easy bijection with standard Young tableaux of the rectangular shape $k \times(n-k)$. By the hook-length formula, their number is $(k(n-k)) ! \prod_{i=0}^{n-k-1} \frac{i !}{(k+i) !}$.

Note, however, $\omega(k, n, d) \neq \omega_{\text {lift }}(k, n, d)$ for $(k, n, d)=(2,5,1)$. Indeed, Galashin pointed out that the monotone path $\{1,2\} \rightarrow\{1,3\} \rightarrow\{1,4\} \rightarrow\{2,4\} \rightarrow\{3,4\}$ cannot be lifted to a rhombus tiling of the the $2 n$-gon $Z(n, 2)$, because it is not weakly separated.

\section{Grassmannian graphs as DuAls of POlyhedral Subdivisions induCED BY PROJECTIONS OF HYPERSIMPLICES}

Let us now discuss the connection between the positive Grassmannian and combinatorics of polyhedral subdivisions. In fact, the positive Grassmannian is directly related to the setup of the previous section for $d=2$.

Theorem 11.1. The poset of complete reduced Grassmannian graphs of type $(k, n)$ ordered by refinement is canonically isomorphic to the Baues poset $\omega(k, n, 2)$ of $\pi$-induced subdivisions for a 2-dimensional cyclic projection $\pi$ of the hypersimplex $\Delta_{k n}$. Under this isomorphism, plabic graphs correspond to tight $\pi$-induced subdivisions and moves of plabic graphs correspond to flips between tight $\pi$-induced subdivisions.

Theorem 4.13(2) ([Pos06, Theorem 13.4]) immediately implies flip-connectivity.

Corollary 11.2. The minimal elements of Baues poset $\omega(k, n, 2)$ are connected by flips.

Example 11.3. The Baues poset $\omega(1, n, 2)$ is the poset of subdivisions of an $n$-gon by non-crossing chords, i.e., it is the Stasheff's associahedron. Its minimal elements correspond to the Catalan number $\frac{1}{n-1}\left(\begin{array}{c}2 n-4 \\ n-2\end{array}\right)$ triangulations of the $n$-gon. 
We can think of the Baues posets $\omega(k, n, 2)$ as some kind of "generalized associahedra." In general, they are not polytopal. But they share some nice features with the associahedron. It is well-known that every face of the associahedron is a direct product of smaller associahedra. The same is true for all $\omega(k, n, 2)$.

Proposition 11.4. For any element $S$ in $\omega(k, n, 2)$, the lower order interval $\left\{S^{\prime} \mid\right.$ $\left.S^{\prime} \leq S\right\}$ in the Baues poset $\omega(k, n, 2)$ is a direct product of Baues posets of the same form $\omega\left(k^{\prime}, n^{\prime}, 2\right)$.

Proof. This is easy to see in terms of complete reduced Grassmannian graphs $G$. Indeed, for any $G$, all refinements of $G^{\prime}$ are obtained by refining all vertices of $G$ independently from each other.

This property is related to the fact that every face of the hypersimplex $\Delta_{k n}$ is a smaller hypersimplex, as we discuss below.

Remark 11.5. Among all reduced Grassmannian/plabic graphs, there is a subset of coherent (or regular) graphs, namely the ones that correspond to the coherent $\pi$-induced subdivisions from $\omega_{\mathrm{coh}}(k, n, 2)$. Each of these graphs can be explicitly constructed in terms of a height function. This subclass depends on a choice of the cyclic projection $\pi$. Regular plabic graphs are related to the study of soliton solutions of Kadomtsev-Petviashvili (KP) equation, see [KoW11, KoW14]. We will investigate the class of regular plabic graphs in GPW].

Let us now give more details on the correspondence between Grassmannian graphs and subdivisions. A cyclic projection $\pi: \Delta_{k n} \rightarrow Q(k, n, 2)$ is the linear map given by a $3 \times n$ matrix $M=\left(u_{1}, \ldots, u_{n}\right)$ such that $\left[u_{1}, \ldots, u_{n}\right] \in G r^{>0}(3, n)$ and $u_{1}, \ldots, u_{n}$ all lie on the same affine plane $H_{1} \subset \mathbb{R}^{3}$. Without loss of generality, assume that $H_{1}=\{(x, y, z) \mid z=1\}$. The positivity condition means that the points $\pi\left(u_{1}\right), \ldots, \pi\left(u_{n}\right)$ form a convex $n$-gon with vertices arranged in the counterclockwise order.

The polytope $Q:=Q(k, n, 2)=\pi\left(\Delta_{k n}\right)$ is the convex $n$-gon in the affine plane $H_{k}=\{(x, y, k)\} \subset \mathbb{R}^{3}$ with the vertices $\pi\left(e_{[1, k]}\right), \pi\left(e_{[2, k+1]}\right), \ldots, \pi\left(e_{[n, k-1]}\right)$ (in the counterclockwise order) corresponding to all consecutive cyclic intervals of size $k$ in $[n]$.

Notice that each face $\gamma$ of the hypersimplex $\Delta_{k n}$ is itself a smaller hypersimplex of the form

$$
\gamma_{I_{0}, I_{1}}:=\left\{\left(x_{1}, \ldots, x_{n}\right) \in \Delta_{k n} \mid x_{i}=0 \text { for } i \in I_{0}, x_{j}=1 \text { for } j \in I_{1}\right\}
$$

where $I_{0}$ and $I_{1}$ are disjoint subsets of $[n]$. So $\gamma \simeq \Delta_{h m}$, where $h=k-\left|I_{0}\right|$ and $m=n-\left|I_{0}\right|-\left|I_{1}\right|$. The projection $\pi$ maps the face $\gamma$ to the $m$-gon $\pi(\gamma)$ that carries an additional parameter $h$.

Thus the $\pi$-induced subdivisions $S$ are in bijective correspondence with the tilings of the $n$-gon $Q$ by smaller convex polygons such that:

(1) Each vertex has the form $\pi\left(e_{I}\right)$ for $I \in\left(\begin{array}{c}{[n]} \\ k\end{array}\right)$.

(2) Each edge has the form $\left[\pi\left(e_{I}\right), \pi\left(e_{J}\right)\right]$ for two $k$-element subsets $I$ and $J$ such that $|I \cap J|=k-1$.

(3) Each face is an $m$-gon of the form $\pi\left(\gamma_{I_{0}, I_{1}}\right)$, as above.

Let $S^{*}$ be the planar dual of such a tiling $S$. The graph $S^{*}$ has exactly $n$ boundary vertices $b_{i}$ corresponding to the sides $\left[\pi\left(e_{[i, i+k-1]}\right), \pi\left(e_{[i+1, i+k]}\right)\right]$ of the $n$-gon $Q$. The internal vertices $v$ of $S^{*}$ (corresponding to faces $\gamma$ of $S$ ) are equipped 
with the parameter $h=h(v) \in\{0, \ldots, \operatorname{deg}(v)\}$. Thus $S^{*}$ has the structure of a Grassmannian graph. Moreover, each face $F$ of $S^{*}$ (corresponding a vertex $\pi\left(e_{I}\right)$ of $S)$ is labelled by a subset $I \in\left(\begin{array}{c}{[n]} \\ k\end{array}\right)$. We can now make the previous theorem more precise.

Theorem 11.6. The map $S \mapsto S^{*}$ is an isomporphism between the Baues poset $\omega(k, n, 2)$ and complete reduced Grassmannian graphs $G$ of type $(k, n)$. For each face $F$ of $G=S^{*}$ corresponding to a vertex $\pi\left(e_{I}\right)$ of $S$, the subset $I \subset\left(\begin{array}{c}{[n]} \\ k\end{array}\right)$ is exactly the face label $I_{F}$ (see Definition 5.2).

Proof. Let us first show that tight $\pi$-induced subdivisions $S$ are in bijection with complete reduced plabic graphs of type $(k, n)$. That means that, in addition to the conditions (1), (2), (3) above, we require the tiling $S$ of the $n$-gon $G$ has all triangular faces. So $S$ is a triangulation of the $n$-gon $Q$ of a special kind, which we call a plabic triangulation.

Such plabic triangulations of the $n$-gon are closely related to plabic tilings from OPS15. The only difference between plabic triangulations and plabic tilings is that the latter correspond not to (3-valent) plabic graphs (as defined in the current paper) but to bipartite plabic graphs. A bipartite plabic graph $G$ is exactly a Grassmannian graph such that each internal vertex either has type $(1, d)$ (white vertex) or type $(d-1, d)$ (black vertex), and every edge of $G$ connects vertices of different colors. Each reduced 3-valent plabic graph $G^{\prime}$ can be easily converted into a bipartite plabic graph $G$ by contrating edges connecting vertices of the same color. It was shown in OPS15, Theorem 9.12] that the planar dual graph of any reduced bipartite plabic graph $G$ can be embedded inside an $n$-gon as a plabic tiling with black and white regions and all vertices of the form $\pi\left(e_{I}\right)$. If we now subdivide the black and white regions of such plabic tiling by chords into triangles, we can get back the plabic triangulation associated with a (3-valent) plabic graph $G^{\prime}$. This shows that any complete reduced (3-valent) plabic graph is indeed the planar dual of a tight $\pi$-induced subdivision.

On the other hand, for each plabic triangulation $S$ we can construct the plabic graph by taking its planar dual $G=S^{*}$ as described above. It is easy to check from the definitions that the decorated strand permutation $w$ of $G$ is exactly $w(i)=i+k$ $(\bmod n)$. It remains to show that this plabic graph $G$ is reduced. Suppose that $G$ is not reduced. Then by Theorem $4.13(5)$, after possibly applying a sequence of moves $(1,4),(2,4)$, and/or $(3,4)$, we get a plabic graph with a pair of parallel edges or with a loop-edge. It is straightforward to check that applying the moves $(1,4)$, $(2,4),(3,4)$ corresponds to local transformations of the plabic triangulation $S$, and transforms it into another plabic triangulations $S^{\prime}$. However, it is clear that if a plabic graph $G$ contains parallel edges of a loop-edge, then the dual graph is not a plabic triangulation. So we get a contradiction, which proves the result for plabic graphs and tight subdivisions.

Now let $G^{\prime}$ be any complete reduced Grassmannian graph of type $(k, n)$, and let $G$ be its plabic refinement. We showed that we can embed the planar dual graph $G^{*}$ as a plabic triangulation $S$ into the $n$-gon. The union of triangles in $S$ that correspond to a single vertex $v$ of $G^{\prime}$ covers a region inside $Q$. We already know that this region is a convex $m$-gon (because we already proved the correspondence for plabic graphs). Thus, for each vertex of $G^{\prime}$, we get a convex polygon in $Q$ and all these polygons form $\pi$-induced subdivision. So we proved that the planar 
dual of $G^{\prime}$ can be embedded as a polyhedral subdivision of $Q$. The inverse map is $S^{\prime} \mapsto G^{\prime}=\left(S^{\prime}\right)^{*}$.

Let us mention a related result of Galashin [Gal16].

Theorem 11.7. Gal16] Complete reduced plabic graphs of type $(k, n)$ are exactly the dual graphs of sections of fine zonotopal tilings of the 3-dimensional cyclic zonotope $Z(n, 3)$ by the hyperplane $H_{k}$.

In view of the discussion above, this result means that any tight $\pi$-induced subdivision in $\omega(k, n, 2)$ can be lifted to a fine zonotopal tiling of the cyclic zonotope $Z(n, 3)$. In other words, the posets $\omega(k, n, 2)$ and $\omega_{\text {lift }}(k, n, 2)$ have the same sets of minimal elements. A natural question to ask: Is the same true for all (not necessarily minimal) elements of $\omega(k, n, 2)$ ?

\section{Membranes and discrete Plateau's problem}

Membranes from the joint project with Lam [LP] provide another related interpretation of plabic graphs. Let $\Phi=\left\{e_{i}-e_{j} \mid i \neq j\right\} \in \mathbb{R}^{n}$, where $e_{1}, \ldots, e_{n}$ are the standard coordinate vectors.

Definition 12.1. [LP A loop $L$ is a closed piecewise-linear curve in $\mathbb{R}^{n}$ formed by line segments $[a, b]$ such that $a, b \in \mathbb{Z}^{n}$ and $a-b \in \Phi$.

A membrane $M$ with boundary loop $L$ is an embedding of a 2-dimensional disk into $\mathbb{R}^{n}$ such that $L$ is the boundary of $M$, and $M$ is made out of triangles $\operatorname{conv}(a, b, c)$, where $a, b, c \in \mathbb{Z}$ and $a-b, b-c, a-c \in \Phi$.

A minimal membrane $M$ is a membrane that has minimal possible area (the number of triangles) among all membranes with the same boundary loop $L$.

The problem about finding a minimal membrane $M$ with a given boundary loop $L$ is a discrete version of Plateau's problem about minimal surface. Informally speaking, membranes correspond to (the duals of) plabic graphs, and minimal membranes correspond to reduced plabic graphs. Here is a more careful claim.

Theorem 12.2. $\left[\mathrm{LP}\right.$ Let $w \in S_{n}$ be a permutation without fixed points with helicity $h(w)=k$. Let $L_{w}$ be the closed loop inside the hypersimplex $\Delta_{k n}$ formed by the line segments $\left[a_{1}, a_{2}\right],\left[a_{2}, a_{3}\right], \ldots,\left[a_{n-1}, a_{n}\right],\left[a_{n}, a_{1}\right]$ such that $a_{i+1}-a_{i}=e_{w(i)}-e_{i}$, for $i=1, \ldots, n$, with indices taken modulo $n$.

Then minimal membranes $M$ with boundary loop $L_{w}$ are in bijection with reduced plabic graphs $G$ with strand permutation w. Explicitly, the correspondence is given as follows. Faces $F$ of $G$ with face labels $I=I_{F}$ correspond to vertices $e_{I}$ of the membrane $M$. Vertices of $G$ with 3 adjacent faces labeled by $I_{1}, I_{2}, I_{3}$ correspond to triangles $\operatorname{conv}\left(e_{I_{1}}, e_{I_{2}}, e_{I_{3}}\right)$ in $M$.

Moves of plabic graphs correspond to local area-preserving transformations of membranes. Any two minimal membranes with the same boundary loop $L_{w}$ can be obtained from each other by these local transformations.

\section{Higher positive Grassmannians and Amplituhedra}

The relation between the positive Grassmannian $G r^{>0}(k, n)$ and the Baues poset $\omega(k, n, 2)$ raises a natural question: What is the geometric counterpart of the Baues poset $\omega(k, n, d)$ for any $d$ ? These "higher positive Grassmannians" should generalize $G r^{>}(k, n)$ in the same sense as Manin-Shekhtman's higher Bruhat orders generalize 
the weak Bruhat order. The first guess is that they might be related to amplituhedra.

Arkani-Hamed and Trnka [AT14, motivated by the study of scattering amplitudes in $\mathcal{N}=4$ supersymmetric Yang-Mills (SYM) theory, defined the amplituhedron $A_{n, k, m}=A_{n, k, m}(Z)$ as the image of the nonnegative Grassmannian $G r \geq(k, n)$ under the "linear projection"

$$
\tilde{Z}: G r^{\geq 0}(k, n) \rightarrow G r(k, k+m), \quad[A] \mapsto\left[A Z^{T}\right]
$$

induced by a totally positive $(k+m) \times n$ matrix $Z$, for $0 \leq m \leq n-k$. The case $m=4$ is of importance for physics.

In general, the amplituhedron $A_{n, k, m}$ has quite mysterious geometric and combinatorial structure. Here are a few special cases where its structure was understood better. For $m=n-k, A_{n, k, n-k}$ is isomorphic to the nonnegative Grassmannian $G r^{\geq 0}(k, n)$. For $k=1, A_{n, 1, m}$ is (the projectivization of) the cyclic polytope $C(n, m)$. For $m=1$, Karp and Williams [KaW16] showed that the structure of the amplituhedron $A_{n, k, 1}$ is equivalent to the complex of bounded regions of the cyclic hyperplane arrangement. In general, the relationship between the amplituhedron $A_{n, k, m}$ and polyhedral subdivisions is yet to be clarified.

\section{REFERENCES}

[ABCGPt16] N. Arkani-Hamed, J. Bourjaily, F. Cachazo, A. Goncharov, A. Postnikov, J. Trnka. Grassmannian Geometry of Scattering Amplitudes. Cambridge University Press, 2016.

[ARS99] C. A. Athanasiadis, J. Rambau, F. Santos. The generalized Baues problem for cyclic polytopes II. In "Geometric combinatorics (Kotor, 1998).". Publ. Inst. Math. (Beograd) (N.S.) 66(80) (1999), 3-15.

[AS02] C. A. Athanasiadis, F. Santos. On the topology of the Baues poset of polyhedral subdivisions. Topology 41 (2002), 423-433.

[Ath01] C. A. Athanasiadis. Zonotopal subdivisions of cyclic zonotopes. Geometriae Dedicata 86 (2001), no. 1-3, 37-57.

[AT14] N. Arkani-Hamed, J. Trnka. The amplituhedron. J. High Energy Phys., (10):33, 2014.

[Bau80] H. J. Baues. Geometry of loop spaces, the cobar construction. Mem. Amer. Math. Soc. 25 (1980) 1-171.

[BFZ05] A. Berenstein, S. Fomin, A. Zelevinsky. Cluster algebras III. Upper bounds and double Bruhat cells. Duke Math. J. 126 (2005), no. 1, 1-52.

[Boh92] J. Bohne. Eine kombinatorische analyse zonotopaler raumaufteilungen. Univ., Diss.Bielefeld, 1992.

[BS92] L.J. Billera, B. Sturmfels. Fiber polytopes. Ann. Math. 135 (1992) 527-549.

[BKS94] L.J. Billera, M.M. Kapranov, B. Sturmfels. Cellular strings on polytopes. Proc. Amer. Math. Soc. 122 (1994) 549-555.

[CK09] S. Chakravarty, Y. Kodama. Soliton solutions of the KP equation and application to shallow water waves. Studies in Applied Mathematics 123 (2009), 83-151.

[CW07] S. Corteel, L. Williams. Tableaux combinatorics for the asymmetric exclusion process. Advances in Applied Math 39 (2007), no. 3, 293-310.

[DKK10] V. I. Danilov, A. V. Karzanov, G. A. Koshevoy. On maximal weakly separated set-systems. J. of Algebraic Combinatorics 32 (2010), no. 4, 497-531.

[FP16] M. Farber, A. Postnikov, Arrangements of equal minors in the positive Grassmannian, Adv. Math. 300 (2016), 788-834.

[FZ99] S. Fomin, A. Zelevinsky. Double Bruhat cells and total positivity. Journal of the American Mathematical Society 12 (1999), no. 2, 335-380.

[FZ02a] S. Fomin, A. Zelevinsky. Cluster algebras. I. Foundations. J. Amer. Math. Soc. 15 (2002), no. 2, 497-529. 
[FZ02b] S. Fomin, A. Zelevinsky. The Laurent phenomenon. Adv. in Appl. Math. 28 (2002), no. $2,119-144$.

[FZ03] S. Fomin, A. Zelevinsky. Cluster algebras. II. Finite type classification. Invent. Math. 154 (2003), no. 1, 63-121.

[FZ07] S. Fomin, A. Zelevinsky. Cluster algebras. IV. Coefficients. Compositio Mathematica 143 (2007), no. 1, 112-164.

[Gal16] P. Galashin. Plabic graphs and zonotopal tilings. arXiv:1611.00492, 2016.

[GGMS87] I. M. Gelfand, R. M. Goresky, R. D. MacPherson, V. V. Serganova. Combinatorial geometries, convex polyhedra, and Schubert cells. Advances in Math. 63 (1987), 301-316.

[GK35] F. R. Gantmacher, M. G. Krein. Sur les matrices oscillatoires. C. R. Acad. Sci. Paris 201 (1935), 577-579.

[GKL17] P. Galashin, S. N. KarP, T. Lam. The totally nonnegative Grassmannian is a ball. arXiv:1707.02010, 2017.

[GKZ94] I. M. Gelfand, M. M. Kapranov, A. V. Zelevinsky. Discriminants, Resultants, and Multidimensional Determinants. Mathematics: Theory \& Applications, Birkhäuser, Boston, 1994.

[GP17] P. Galashin, A. Postnikov. Purity and separation for oriented matroids. arXiv:1708.01329, 2017.

[GPW] P. Galashin, A. Postnikov, L. Williams. Regular plabic graphs. In preparation.

[KLS13] A. Knutson, T. Lam, D. Speyer. Positroid varieties: juggling and geometry. Compositio Mathematica 149 (2013), no. 10, 1710-1752.

[KaW16] S. N. KARP, L. Williams. The $m=1$ amplituhedron and cyclic hyperplane arrangements. arXiv: $1608.08288,2016$.

[KoW11] Y. Kodama, L. Williams. KP solitons, total positivity, and cluster algebras. Proceedings of the National Academy of Sciences, (2011) 108 (22), 8984-8989.

[KoW14] Y. Kodama, L. Williams. KP solitons and total positivity on the Grassmannian. Inventiones mathematicae 198 (2014), no. 3, 637-699.

[Liu16] G. LiU. A counterexample to the extension space conjecture for realizable oriented matroids. arXiv:1606.05033 2016.

[Lam15] T. LAM. Totally nonnegative Grassmannian and Grassmann polytopes, arXiv:1506.00603, 2015.

[LP] T. LAM, A. Postnikov. Polypositroids I. In preparation.

[Lus90] G. Lusztig. Canonical bases arising from quantized enveloping algebras. J. Amer. Math. Soc. 3 (1990), no. 2, 447-498.

[Lus92] G. Lusztig. Canonical bases in tensor products. Proc. Nat. Acad. Sci. U.S.A. 89 (1992), no. 17, 8177-8179.

[Lus93] G. LuszTig, Introduction to quantum groups, Progress in Mathematics, vol. 110, Birkhäuser, Boston, 1993.

[Lus94] G. LuszTig. Total positivity in reductive groups. Lie Theory and Geometry: In Honor of B. Kostant, Progress in Mathematics, vol. 123, Birkhäuser, Boston, 1994, pp. 531568.

[Lus98a] G. LuszTig. Total positivity in partial flag manifolds. Representation Theory, 2 (1998), 70-78.

[Lus98b] G. Lusztig. Introduction to total positivity. Positivity in Lie theory: open problems, 133-145, de Gruyter Expositions in Mathematics, 26, de Gruyter, Berlin, 1998.

[LZ98] B. LeClerc, A. Zelevinsky. Quasicommuting families of quantum Plücker coordinates. American Mathematical Society Translations, Ser. 2 181, 1998.

[Mnë88] N. E. MNËv. The universality theorems on the classification problem of configuration varieties and convex polytope varieties. pp. 527-543, in "Topology and Geometry: Rohlin Seminar," O. Ya. Viro ed., Lecture Notes in Mathematics 1346, SpringerVerlag, Berlin, 1988.

[MaS86] Yu. I. Manin, V. V. Shekhtman. Higher Bruhat orderings connected with the symmetric group. Funktsional. Anal. i Prilozhen., 20(2):74-75, 1986.

[MuS16] G. Muller, D. E. Speyer, The twist for positroid varieties, arXiv:1606.08383 2016.

[Oh11] S. Он. Positroids and Schubert matroids. J. Combin. Theory Ser. A 118 (2011), no. 8, $2426-2435$. 
[OPS15] S. Оh, A. Postnikov, D. E. Speyer. Weak separation and plabic graphs. Proc. Lond. Math. Soc. (3) 110 (2015), no. 3, 721-754.

[Pos06] A. Postnikov. Total positivity, Grassmannians, and networks. arXiv:math/0609764. 2006.

[PSW09] A. Postnikov, D. Speyer, L. Williams. Matching polytopes, toric geometry, and the non-negative part of the Grassmannian. Journal of Algebraic Combinatorics 30 (2009), no. 2, 173-191.

[Ram97] J. Rambau. Triangulations of cyclic polytopes and higher Bruhat orders, Mathematika 44 (1997), no. 1, 162-194.

[Rei99] V. REINER. The generalized Baues problem. In New perspectives in algebraic combinatorics (Berkeley, CA, 1996-97), 293-336, Math. Sci. Res. Inst. Publ., volume 38, Cambridge Univ. Press, Cambridge, 1999.

[Rie98] K. Rietsch. Total Positivity and Real Flag Varieties, Ph.D. Thesis, M.I.T. 1998.

[Rie99] K. Rietsch. An algebraic cell decomposition of the nonnegative part of a flag variety, Journal of Algebra 213 (1999), no. 1, 144-154.

[RS00] J. Rambau, F. Santos. The generalized Baues problem for cyclic polytopes I. European Journal of Combinatorics 21 (2000), no. 1, Pages 65-83.

[RW10] R. RiEtsch, L. Williams. Discrete Morse theory for totally non-negative flag varieties. Advances Math. 223 (2010), no. 6, 1855-1884.

[RZ96] J. Rambau, G. M. Ziegler. Projections of polytopes and the generalized Baues conjecture. Discrete Comput. Geom. 16 (1996) 215-237.

[Sco05] J. S. SCOTT. Quasi-commuting families of quantum minors. J. Algebra 290 (2005), no. $1,204-220$.

[Sco06] J. S. ScotT. Grassmannians and cluster algebras. Proc. London Math. Soc. (3) 92 (2006), no. 2, 345-380.

[Sch30] I. J. Schoenberg. Uber variationsvermindende lineare Transformationen. Math. Z. 32 (1930), 321-328.

[SZ93] B. Sturmfels, G. M. Ziegler, Extension spaces of oriented matroids. Discrete Comput. Geom. 10 (1993), 23-45.

[Tal08] K. TAlaskA. A formula for Plücker coordinates associated with a planar network. Int. Math. Res. Not. IMRN 2008, 19 pp.

[VK91] V. A. Voevodskit, M. M. Kapranov. The free n-category generated by a cube, oriented matroids and higher Bruhat orders. Funktsional. Anal. i Prilozhen., 25(1):6265, 1991.

[Zie93] G. M. ZiEgler. Higher Bruhat orders and cyclic hyperplane arrangements. Topology, 32(2):259-279, 1993.

Department of Mathematics, Massachusetts Institute of Technology, 77 Massachusetts Avenue, Cambridge MA 02139

E-mail address: apost@math.mit.edu 